\title{
Chapter 16 \\ From Computer Time to Legal Civil Time: IANA tz, IETF tzdist, etc.
}

\author{
Steve Allen
}

\begin{abstract}
The internal time scale in computing and telecommunication systems is often based on UTC, but users of systems expect to see the local civil or legal time in their jurisdiction. Currently there are about 40-80 different time zones in active use. Historically there have been around 200 sets of rules needed to compute the differences between UT and local time. These rule sets have been painstakingly maintained by international agencies and corporations, and they are typically distributed to machines via updates to operating systems. Unfortunately, jurisdictions have changed their rules with little or no notice, and some machines do not get operating system updates in a timely fashion or ever. These lacunae are motivations for ongoing efforts among international standard organizations and corporations.
\end{abstract}

Keywords tzdist • Timezones • Operating systems • International Atomic Time (TAI) • Coordinated Universal Time (UTC) • Civil time • Legal time • Application programming interfaces (APIs)

The internal time scale in computing and telecommunication systems is often based on UTC, but users of systems expect to see the local civil or legal time in their jurisdiction. Currently there are about 40-80 different time zones in active use. Historically there have been around 200 sets of rules needed to compute the differences between UT and local time. These rule sets have been painstakingly maintained by international agencies and corporations, and they are typically distributed to machines via updates to operating systems. Unfortunately, jurisdictions have changed their rules with little or no notice, and some machines do not get operating system updates in a timely fashion or ever. These lacunae are motivations for ongoing efforts among international standard organizations and corporations.

\footnotetext{
S. Allen $(\bowtie)$

University of California Observatories/Lick Observatory, ISB 260, 1156 High Street,

Santa Cruz, CA 95064, USA

e-mail: sla@ucolick.org
} 